\title{
Delineamentos aumentados no melhoramento de plantas em condições de restrições de recursos
}

\author{
Augmented designs in plant breeding under resource constrain
}

\author{
Luiz Alexandre Peternelli' Emanuel Fernando Maia de Souza ${ }^{\text {II }}$ \\ Márcio Henrique Pereira Barbosa ${ }^{\mathrm{III}}$ Melissa Pisaroglo de Carvalho ${ }^{\mathrm{IV}}$
}

RESUMO

Este trabalho teve por objetivo comparar a eficiência na seleção de genótipos e a qualidade das estimativas dos componentes de variância e da herdabilidade, empregando o delineamento em blocos aumentados (DBA), o delineamento em blocos aumentados duplicados (DBAD) e o grupo de experimentos em blocos casualizados com tratamentos comuns (EBCTC). Para a comparação, foi imposta a condição de que o número de genótipos sob seleção era maior do que o número de unidades experimentais disponíveis para os delineamentos com repetição. Quatro cenários foram compostos da combinação de diferentes valores de herdabilidade e do coeficiente de variação residual paramétricos. Das 2.400 simulações por cenário, o DBA apresentou a maior eficiência de seleção. Os delineamentos com repetição (DBAD e EBCTC) apresentaram eficiência de seleção semelhante entre si. É possível concluir que a não realização de repetições, avaliandose um maior número de genótipos, pode trazer melhores resultados ao programa de melhoramento de plantas.

Palavras-chave: seleção, simulação, Saccharum spp.

\section{ABSTRACT}

This study aimed to compare the efficiency in the selection of genotypes and the quality of the estimates of the variance components and heritability using the augmented block designs $(A B D)$, the duplicated augmented block designs $(D A B D)$ and the group of randomized blocks design experiments with common treatments (ERBCT). For comparison, it was imposed a condition that the number of genotypes under selection was greater than the number of units available for experimental designs with repetition. Four scenarios were composed of a combination of different values of heritability and parametric residual coefficient of variation. From the 2400 simulations per scenario the $A B D$ had the highest selection efficiency. The designs with repetition (DABD and ERBCT) had similar selection efficiency between them. It is possible to conclude that not using repetitions, and therefore, evaluating a larger number of genotypes, can bring better results to the plant breeding program.

Key words: selection, simulation, Saccharum spp.

\section{INTRODUÇÃO}

O sucesso de um programa de melhoramento está intimamente ligado à avaliação de um grande número de genótipos (HOGARTH \& ALLSOPP, 2000; PETERNELLI \& BARBOSA, 2004). No caso da canade-açúcar, a avaliação de genótipos nas fases iniciais torna-se difícil, pois nem sempre é possível implementar um experimento completo e/ou balanceado, uma vez que podem ocorrer restrições financeiras e físicas, como mão-de-obra, área experimental e material propagativo (SOUZA et al., 2000).

Os melhoristas têm buscado alternativas que contornem tais situações, como o uso de experimentos incompletos ou de delineamentos aumentados (SOUZA et al., 2000, 2003; MATSUOKA et al., 2005; RESENDE et al., 2006). Entre essas opções, o uso de delineamentos aumentados é atrativo, pois, no caso de falta de material propagativo, os tratamentos podem ser testados com apenas uma repetição (FEDERER, 1956).

\footnotetext{
'Departamento de Estatística, Universidade Federal de Viçosa (UFV), 36570-000, Viçosa, MG, Brasil. E-mail: peternelli@ufv.br. Autor para correspondência.

IIDepartamento de Agronomia, Universidade Federal de Rondônia (UNIR), Rolim de Moura, RO, Brasil.

IIIDepartamento de Fitotecnia, UFV, Viçosa, MG, Brasil.

${ }^{\text {IV }}$ Programa de Pós-graduação em Genética e Melhoramento, UFV, Viçosa, MG, Brasil.
} 
Nos programas de melhoramento de canade-açúcar, são utilizados também o grupo de experimentos em blocos casualizados com tratamentos comuns e o delineamento em blocos aumentados duplicados. Este último oferece a vantagem de ser menos trabalhoso no momento da instalação em campo (PETERNELLI\& BARBOSA, 2004; MATSUOKA etal., 2005).

Várias propostas de métodos de análises a serem empregados nos delineamentos aumentados ou de experimentos incompletos estão presentes na literatura (FEDERER, 1956; DUARTE et al., 2001; DUARTE \& VENCOVSKY, 2001, 2005; SANTOS etal., 2002; SOUZA et al., 2006). Os principais resultados se resumem no uso de modelos mistos e na escolha do método de estimação dos componentes de variância, que deve ser apropriado para cada situação. Em alguns trabalhos, encontra-se a proposta de se avaliar a real eficiência de seleção dos melhores genótipos entre os diferentes delineamentos de uso potencial, em que é evidenciado que o uso de repetições é favorável quando não há restrições de recursos (SANTOS et al., 2002; SOUZA et al., 2006).

Contudo, restam dúvidas a respeito da eficiência desses delineamentos, principalmente se não houver recursos físicos ou financeiros suficientes para se instalar um experimento com repetições para todos os genótipos disponíveis. A avaliação comparativa desses delineamentos tem sido pouco explorada na literatura. Assim, este trabalho foi realizado visandose a avaliar a eficiência de seleção e da estimação dos componentes de variância em condições de restrições de recursos, onde a área experimental disponível é fixa, e variam-se o número de tratamentos a serem testados de acordo com o delineamento escolhido.

\section{MATERIAIS E MÉTODOS}

As simulações foram baseadas no esquema hipotético de um grupo de experimentos em blocos casualizados com tratamentos comuns (EBCTC), constituindo 138 unidades experimentais (Figura 1). Foram considerados três tratamentos comuns, repetidos em todos os blocos, e 120 tratamentos regulares (novos genótipos) que podem ou não ser repetidos nos experimentos. Sob a condição de área experimental limitada, optou-se por duas situações: i) avaliar os 120 tratamentos no Delineamento em Blocos Aumentados (DBA) sem repetições, ou ii) avaliar 60 genótipos pelo EBCTC ou delineamento em Blocos Aumentados Duplicados (DBAD), ou seja, realizandose duas repetições e, dessa forma, podendo-se absorver, por vez, apenas 60 genótipos. Portanto, no caso ii, seriam descartados 60 genótipos dentre os 120 disponíveis. Para a escolha dos genótipos a serem alocados quando se opta pelo EBCTC ou DBAD, foi realizada uma amostragem aleatória simples de 60 tratamentos regulares dentre os 120 disponíveis.

Foram gerados quatro cenários a partir do modelo estatístico adotado para o EBCTC (modelo 1), descrito posteriormente neste trabalho. Os parâmetros estipulados seguiram os propostos por SOUZA et al. (2006). Para a geração de dados, foram estipulados dois coeficientes de variação $(\mathrm{CV}), 10$ e $20 \%$, para cada um dos erros, o residual e o entre experimentos. O CV para blocos dentro de experimento foi igual a $10 \%$, enquanto que a média geral foi fixada em 70 unidades. $O$ valor de herdabilidade $\left(\mathrm{h}^{2}\right)$ para os tratamentos comuns foi igual a 0,8 . Para os tratamentos regulares, optou-se por avaliar dois valores de $h^{2}: 0,7$ e 0,3 . Foram gerados 2.400 conjuntos de dados para cada cenário, que foram analisados segundo os métodos apresentados a seguir.

Métodos de análise para o EBCTC podem ser encontrados em GOMES (1987) e em RAMALHO et al. (2000). O seguinte modelo estatístico foi utilizado: $\mathrm{Y}_{\mathrm{ijk}}=\mu+\mathrm{E}_{\mathrm{i}}+\mathrm{B}_{(\mathrm{ijj}}+\mathrm{G}_{\mathrm{k}}+\mathrm{e}_{\mathrm{ijk}}$ (modelo 1), em que: $\mathrm{Y}_{\mathrm{ijk}}=$ valor observado do tratamento $\mathrm{k}$, no bloco $\mathrm{j}$, do experimento $\mathrm{i} ; \mathrm{E}_{\mathrm{i}}=$ efeito do experimento $\mathrm{i}, \mathrm{i}=1,2,3$; $\mathrm{B}_{\text {(i)j }}=$ efeito do bloco $\mathrm{j}, \mathrm{j}=1,2$, dentro do experimento $\mathrm{i}$; $\mathrm{G}_{\mathrm{k}}=\mathrm{T}_{\mathrm{k}}, \mathrm{T}_{(\mathrm{j}) \mathrm{k}}$, sendo $\mathrm{G}_{\mathrm{k}}$ o efeito de tratamento

\begin{tabular}{|c|c|c|c|}
\hline \multirow[b]{2}{*}{ Rep 1} & Experimento 1 & Experimento 2 & Experimento 3 \\
\hline & $\begin{array}{r}\mathrm{A}, \mathrm{B}, \mathrm{C}+ \\
1,2, \ldots 20\end{array}$ & $\begin{array}{c}\mathrm{A}, \mathrm{B}, \mathrm{C}+ \\
21,22, \ldots 40\end{array}$ & $\begin{array}{c}\text { A, B, C + } \\
41,42, \ldots 60\end{array}$ \\
\hline Rep 2 & $\begin{array}{l}\mathrm{A}, \mathrm{B}, \mathrm{C}+ \\
1,2, \ldots 20\end{array}$ & $\begin{array}{c}\mathrm{A}, \mathrm{B}, \mathrm{C}+ \\
21,22, \ldots 40\end{array}$ & $\begin{array}{c}\text { A, B, C+ } \\
41,42, \ldots 60\end{array}$ \\
\hline gura & $\begin{array}{l}\text { Esquema de um grupo } \\
\text { repetições por experim } \\
\text { regulares, todos alocad }\end{array}$ & $\begin{array}{l}\text { em blocos casualiz: } \\
\text { esentam os tratamer } \\
\text { dentro dos blocos }\end{array}$ & $\begin{array}{l}\text { mentos comuns, com } \\
\text { os números os tratame }\end{array}$ \\
\hline
\end{tabular}

Ciência Rural, v.39, n.9, dez, 2009. 
(modificado por SCOTT \& MILLIKEN, 1993; DUARTE et al., 2001), $\mathrm{T}_{\mathrm{k}}$ : efeito fixo do tratamento comum, $\mathrm{k}^{\prime}=1$, 2,$3 ; \mathrm{T}_{(\mathrm{j}) \mathrm{k}}:$ efeito aleatório do tratamento regular $\mathrm{k}$ dentro do bloco j, sendo $\mathrm{k}=4,5, \ldots, 63 ; \therefore \mathrm{T}_{(\mathrm{j}) \mathrm{k}} \sim N I D\left(0, \sigma_{\mathrm{T}(\mathrm{j}) \mathrm{k}}^{2}\right)$; $\mathrm{e}_{\mathrm{ij \textrm {k }}}=$ efeito aleatório do erro experimental.

Devido à disposição peculiar das unidades experimentais no campo (Figura 1), pode-se considerar, alternativamente, no sentido horizontal, a ocorrência de dois experimentos em blocos aumentados com três blocos (em que cada experimento i seria, agora, considerado bloco j), dispostos lado a lado. O conjunto desses dois DBA formaria um DBAD, em que a primeira repetição do DBAD corresponderia ao conjunto dos primeiros blocos adjacentes (Rep.1), e a outra ao conjunto dos blocos restantes (Rep.2). Nesse caso, os dados de cada repetição (ou seja, cada DBA) são submetidos inicialmente à análise de variância intrabloco: $\mathrm{Y}_{\mathrm{kj}}=\mu+\mathrm{T}_{\mathrm{k}}, \mathrm{T}_{(\mathrm{j}) \mathrm{k}}+\mathrm{B}_{\mathrm{j}}+\mathrm{e}_{\mathrm{kj}}$ (modelo 2), em que: $Y_{\mathrm{kj}}$ : valor observado na parcela experimental do bloco $\mathrm{j}$, que recebeu o tratamento regular $\mathrm{k}$ ou o tratamento comum k' dentro do bloco j; $\mu$ : média geral; $\mathrm{T}_{\mathrm{k}}$ : efeito fixo do tratamento comum, $\mathrm{k}^{\prime}=1,2,3 ; \mathrm{T}_{(\mathrm{j}) \mathrm{k}}$ : efeito aleatório do tratamento regular $\mathrm{k}$ dentro do bloco $\mathrm{j}$, sendo $\mathrm{k}=4,5, \ldots, \mathrm{r}+\mathrm{c}$, em que $\mathrm{r}$ é o número de tratamentos regulares considerados e $\mathrm{c}$ é o número de tratamentos comuns; $\therefore \mathrm{T}_{(\mathrm{j}) \mathrm{k}} \sim N I D\left(0, \sigma_{\mathrm{T}(\mathrm{j}) \mathrm{k}}^{2}\right) ; \mathrm{B}_{\mathrm{j}}$ : efeito do bloco $\mathrm{j}$, sendo $\mathrm{j}=1,2, . ., \mathrm{b}$, em que $\mathrm{b}$ é o número de blocos; e $\mathrm{e}_{\mathrm{kj}}$ : efeito aleatório do erro experimental.

Posteriormente, para cada repetição (correspondente a um particular DBA), são obtidas, a partir da análise intrabloco, as médias ajustadas dos tratamentos regulares. Essas médias foram utilizadas para compor uma nova análise de variância, excluindo os c tratamentos comuns, seguindo-se o modelo: $\mathrm{Y}_{\mathrm{kj}}=$ $\mu+\mathrm{T}_{\mathrm{k}}+\mathrm{R}_{\mathrm{j}}+\mathrm{e}_{\mathrm{kj}}$ (modelo 3), em que: $\mathrm{Y}_{\mathrm{kj}}$ : média ajustada da parcela experimental do tratamento $\mathrm{k}(\mathrm{k}=1,2, \ldots, 60)$ da repetição $\mathrm{j} ; \mu$ : média geral; $\mathrm{R}_{\mathrm{j}}$ : efeito da repetição $\mathrm{j}$, sendo $\mathrm{j}=1 \mathrm{e} 2 ; \mathrm{T}_{\mathrm{k}}$ : efeito aleatório do tratamento $\mathrm{k} ; \therefore \mathrm{T}_{\mathrm{k}} \sim$ $N I D\left(0, \sigma^{2}{ }_{\mathrm{Tk}}\right) ; \mathrm{e}_{\mathrm{k}}:$ efeito aleatório do erro experimental. Para o DBA, foram considerados seis blocos e 120 tratamentos regulares (adaptado da Figura 1). A sua análise foi realizada a partir do modelo 2, usando-se $\mathrm{b}=6$ e $\mathrm{r}=120$.

Para cada um dos 2.400 conjuntos de dados simulados, após a obtenção das médias ajustadas dos tratamentos regulares de cada delineamento, foram selecionados seis ou 12 novos genótipos com as maiores estimativas da variável avaliada. Na prática, essa quantidade selecionada corresponderia, para o caso do DBA, a uma seleção de 5 ou $10 \%$ dos genótipos, respectivamente; e, para o caso do DBAD e do EBCTC, a uma seleção de 10 ou $20 \%$ dos genótipos, respectivamente. Nas simulações, como eram conhecidos os verdadeiros tratamentos superiores
(REAL), ou seja, de maiores valores paramétricos das médias ou efeitos de tratamentos, foi possível avaliaremse as porcentagens de coincidência entre os tratamentos selecionados pelos delineamentos e o REAL. As avaliações de eficiência na seleção foram realizadas considerando-se somente os tratamentos regulares. Os resultados encontrados também foram comparados com aqueles obtidos caso fosse realizada uma seleção aleatória simples de seis ou 12 genótipos, segundo a estrutura de cada delineamento.

Os componentes de variância foram estimados pelo método de máxima verossimilhança restrita (REML). A qualidade da estimação foi medida pela acurácia relativa média (ARM) ou viés relativo médio, em porcentagem, em que $\mathrm{ARM}=\sum_{i=1}^{N}\left(\frac{\hat{\theta}_{i}-\theta}{\theta}\right) \cdot 100 / N$, sendo $N$ : o número total de experimentos; $\theta$ : o valor paramétrico de interesse; e $\hat{\theta}$ : o estimador de $\theta$. Acurácia indica o quão próximo do valor real está o valor calculado (SOKALE ROHLF, 1981).

Todas as funções, algoritmos de simulação e análises estatísticas foram realizadas empregando-se o programa livre R (R DEVELOPMENT CORE TEAM, 2008).

\section{RESULTADOS E DISCUSSÕES}

Nas condições do presente trabalho, e desconsiderando-se variações devidas às diferentes herdabilidades e variâncias residuais, ou seja, agrupando-se os 2.400 resultados das simulações de cada cenário, pode-se observar que as distribuições da porcentagem de coincidência para o EBCTC e o DBAD são semelhantes (Figura 2). Resultados equivalentes no uso desses dois delineamentos também foram encontrados por SOUZA et al. (2006) na situação de não haver restrição de recursos. Com o aumento no número de genótipos selecionados, ocorreu um aumento na porcentagem de coincidência dos selecionados com o REAL (Tabela 1 e Figura 2). Esse resultado seria esperado, uma vez que, num caso extremo, se fosse possível juntar nos experimentos todos os genótipos disponíveis para avaliação, e se todos fossem selecionados, a porcentagem de coincidência entre os melhores selecionados e os melhores de fato (REAL) seria igual a $100 \%$.

Em ambos os níveis de seleção, comparando-se os delineamentos, a distribuição de coincidências do DBA apresenta, em média, maiores valores. Todavia, em todas as situações observa-se que as porcentagens de coincidência são inferiores a $53 \%$. 



Figura 2 - Distribuição da porcentagem de coincidência entre os verdadeiros reais genótipos superiores e os genótipos selecionados pelo delineamento em blocos aumentados (DBA), pelo delineamento em blocos aumentados duplicados (DBAD) e pelo grupo de experimentos em blocos casualizados com tratamentos comuns (EBCTC) na seleção de seis (a) ou 12 (b) novos genótipos, de um total de 120, após agruparem-se as 9.600 simulações. O asterisco representa a média aritmética.

As seleções dos genótipos baseadas no uso dos delineamentos resultaram em estimativas de coincidência maiores do que aquelas obtidas por uma simples seleção ao acaso de seis ou 12 genótipos (Tabela 1). Os valores exatos da porcentagem de coincidência podem ser obtidos calculando-se, inicialmente, o valor esperado da variável aleatória $X=$ \{número de coincidências entre os indivíduos selecionados via uso dos delineamentos e os REAIS \} com base na distribuição hipergeométrica de $\mathrm{X}$ em amostras de tamanho seis ou 12, do total de 60 ensaiados (caso do EBCTC e do DBAD), ou do total de 120 ensaiados (caso do DBA). Posteriormente, o valor esperado da v.a. X seria transformado em porcentagem de acordo com o número de indivíduos selecionados. Nesse caso, os valores esperados da porcentagem de coincidência seriam iguais a 5 e $10 \%$, respectivamente, para o total de seis ou 12 selecionados. Esse resultado enfatiza a vantagem de, já nas fases iniciais, organizarem-se os tratamentos segundo algum delineamento.

O DBA apresentou maior eficiência (cerca de seis ou nove vezes maior que o acaso contra cerca de duas vezes maior que o acaso para os outros delineamentos) quando a seleção é de seis genótipos (Tabela 1). Essa eficiência reduz-se um pouco ao se realizar a seleção de 12 genótipos.

Tabela 1 - Porcentagem de coincidência média entre os verdadeiros reais genótipos superiores (REAIS) e os genótipos selecionados pelo delineamento em blocos aumentados (DBA), pelo delineamento em blocos aumentados duplicados (DBAD), pelo grupo de experimentos em blocos casualizados com tratamentos comuns (EBCTC) e ao acaso, em 2.400 simulações por cenário.

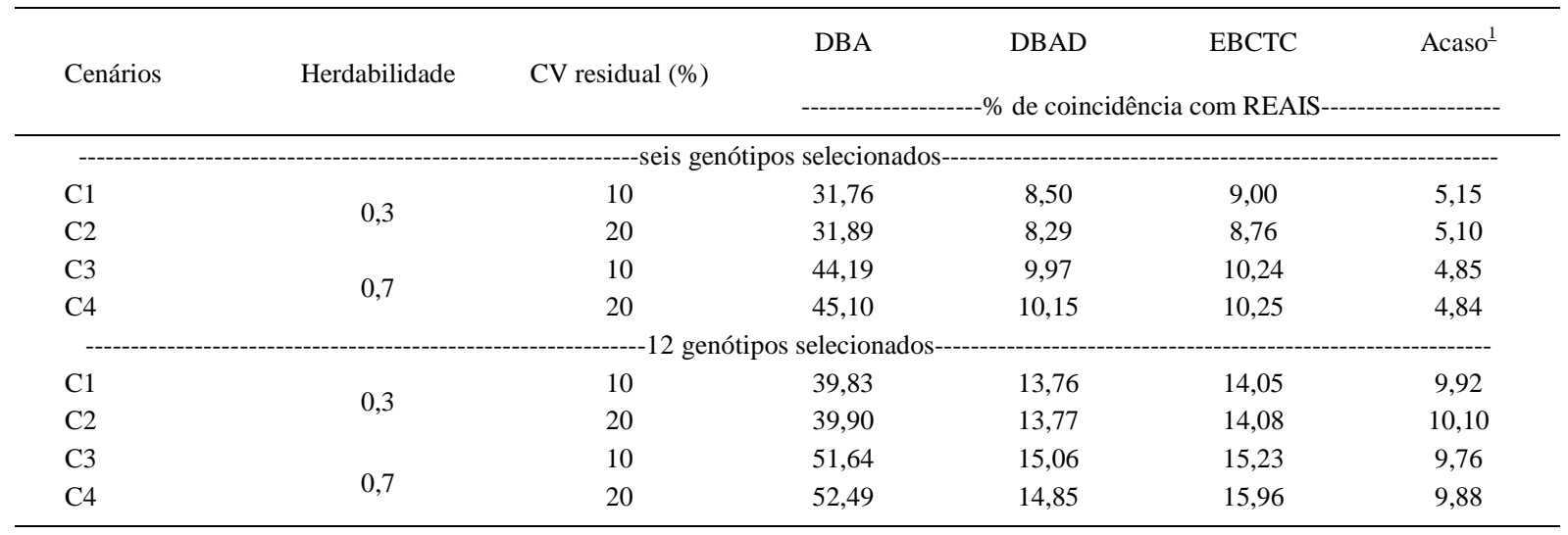

1 Valores percentuais referentes ao número de coincidências entre os seis ou 12 indivíduos ordenados via delineamentos e os seis ou 12 melhores de fato ("REAIS”). 
Comparando-se os delineamentos, pode-se observar que o DBA foi o que apresentou maiores estimativas médias de coincidência com os REAIS, ou seja, maior eficiência de seleção. As maiores eficiências de seleção ocorrem para os cenários em que a variável apresenta maior valor de herdabilidade (Tabela 1). O DBA apresentou os maiores ganhos de eficiência de seleção com o aumento da herdabilidade (aproximadamente, $40 \%$ na porcentagem de coincidência com o REAL). Por outro lado, a redução na precisão do experimento, dada pelo aumento do valor paramétrico do coeficiente de variação residual, não alterou os valores de eficiência de seleção. Nos casos em que se optou por descartar $50 \%$ dos tratamentos para se instalar os experimentos que garantem o princípio experimental da repetição (EBCTC e DBAD), houve perda de eficiência de seleção, quando comparados com a não realização da repetição. Portanto, dada essa situação, recomenda-se empregar o delineamento em blocos aumentados com todos os tratamentos.

A estimação dos componentes de variância e de $\mathrm{h}^{2}$ foi influenciada pela escolha do delineamento (Tabela 2). Em geral, o DBAD tende a subestimar os componentes de variância e a $\mathrm{h}^{2}$, apresentando valores médios negativos para ARM. Adicionalmente, o DBAD apresenta a maior parte das estimativas menos precisas, ou seja, com maiores valores absolutos para a ARM. Para o DBA e o EBCTC, as estimativas de $h^{2}$ apresentaram um comportamento similar. Nos casos de menor valor de $\mathrm{h}^{2}$, houve uma tendência em se observar valores superestimados, enquanto que, nos casos de maior $\mathrm{h}^{2}$, foram observados valores subestimados. Contudo, deve-se observar que os valores médios foram próximos do valor paramétrico. Esses resultados concordam com os de SOUZA et al. (2000 e 2006) e com os de BEARZOTI et al. (1997). Tais autores afirmam que o delineamento em blocos aumentados tende a apresentar discrepâncias nos valores estimados de $\mathrm{h}^{2}$ quando comparados a outros delineamentos.

Considerando-se apenas a qualidade da estimação dos componentes de variância (Tabela 2), o emprego do EBCTC ou do DBA proporcionou estimativas menos tendenciosas para os parâmetros avaliados. A superestimação da variância residual para o DBA era esperada. FEDERER (1956), quando propôs os delineamentos aumentados, chamou atenção para as maiores estimativas da variação residual do delineamento em relação a um delineamento completo.

Tabela 2 - Valores médios e acurácia relativa média (ARM) dos componentes de variância e herdabilidade estimados pelo delineamento em blocos aumentados (DBA), pelo delineamento em blocos aumentados duplicados (DBAD), pelo grupo de experimentos em blocos casualizados com tratamentos comuns (EBCTC) e seus valores paramétricos $(\theta)$. 2.400 simulações por cenário.

\begin{tabular}{|c|c|c|c|c|c|c|c|c|}
\hline & \multicolumn{2}{|c|}{ Cenário C1 } & \multicolumn{2}{|c|}{ Cenário C2 } & \multicolumn{2}{|c|}{ Cenário C3 } & \multicolumn{2}{|c|}{ Cenário C4 } \\
\hline \multirow[t]{2}{*}{$\theta$} & \multicolumn{2}{|c|}{------------21,00------------ } & \multicolumn{2}{|c|}{------------84,00------------ } & \multicolumn{2}{|c|}{-----------114,33----------- } & \multicolumn{2}{|c|}{-----------457,33----------. } \\
\hline & média & ARM & média & ARM & média & ARM & média & ARM \\
\hline DBA & 22,07 & 43,97 & 94,35 & 51,51 & 112,31 & 9,78 & 482,70 & 17,86 \\
\hline DBAD & 1,74 & $-79,97$ & 7,52 & $-79,98$ & 17,35 & $-76,53$ & 68,88 & $-77,44$ \\
\hline ЕВСТC & 32,22 & 80,10 & 127,25 & 75,07 & 132,53 & 16,35 & 496,41 & 16,15 \\
\hline \multirow[t]{2}{*}{$\theta$} & \multicolumn{2}{|c|}{------------49,00------------ } & \multicolumn{2}{|c|}{-196,00- } & \multicolumn{2}{|c|}{------------49,00------------- } & \multicolumn{2}{|c|}{ 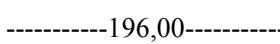 } \\
\hline & média & ARM & média & ARM & média & ARM & média & ARM \\
\hline DBA & 46,95 & 8,15 & 181,13 & 4,39 & 49,63 & 15,85 & 196,05 & 15,29 \\
\hline DBAD & 6,58 & $-81,29$ & 27,61 & $-80,68$ & 13,55 & $-66,43$ & 55,25 & $-66,15$ \\
\hline EBCTC & 48,92 & 2,72 & 127,25 & 1,75 & 49,13 & 3,16 & 197,03 & 3,21 \\
\hline \multirow[t]{2}{*}{$\theta$} & \multicolumn{2}{|c|}{----------0,30---------- } & \multicolumn{2}{|c|}{------ } & \multicolumn{2}{|c|}{ 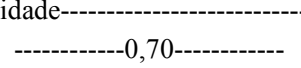 } & \multicolumn{2}{|c|}{------------0,70-----------. } \\
\hline & média & ARM & média & ARM & média & ARM & média & ARM \\
\hline DBA & 0,38 & 25,74 & 0,39 & 31,24 & 0,65 & $-6,55$ & 0,67 & $-4,60$ \\
\hline DBAD & 0,21 & $-29,48$ & 0,21 & $-29,48$ & 0,50 & $-29,00$ & 0,50 & $-28,45$ \\
\hline EBCTC & 0,39 & 29,99 & 0,39 & 30,01 & 0,69 & $-1,78$ & 0,69 & $-1,44$ \\
\hline
\end{tabular}

${ }^{(1)}$ Os cenários C1, C3 e C2, C4 correspondem, respectivamente, aos valores de 10 e 20\% do coeficiente de variação residual. 


\section{CONCLUSÕES}

Quando houver restrição de área, é preferível avaliar mais genótipos empregando-se o delineamento em blocos aumentados (DBA) do que reduzir o número de genótipos regulares utilizando-se repetição desses em delineamentos como blocos aumentados duplicados (DBAD) ou grupos de experimentos em blocos casualizados com tratamentos comuns (EBCTC). O DBAD proporcionou as estimativas menos acuradas para os componentes de variância e herdabilidade em relação ao DBA e ao EBCTC.

\section{AGRADECIMENTOS}

Ao Conselho Nacional de Desenvolvimento Científico e Tecnológico $(\mathrm{CNPq})$, pela bolsa de pesquisa e estudo, e à Fundação de Amparo à Pesquisa do Estado de Minas Gerais (FAPEMIG), pelo apoio financeiro, o que permitiu a compra de equipamentos e o desenvolvimento deste trabalho.

\section{REFERÊNCIAS}

BEARZOTI, E. et al. Comparação entre métodos estatísticos na avaliação de clones em um programa de melhoramento de batata. Pesquisa Agropecuária Brasileira, v.32, p.877884, 1997. Disponível em: <http://webnotes.sct.embrapa.br/ $\mathrm{pab} / \mathrm{pab} . \mathrm{nsf} / \mathrm{ecd} 4 \mathrm{ca} 3 \mathrm{ff} 88 \mathrm{efcfa} 032564 \mathrm{~cd} 004$ ea $083 /$ d28013a28179f86c0325650c00661241? OpenDocument>. Acesso em: 24 jul. 2009.

DUARTE, J.B.; VENCOVSKY, R. Estimação e predição por modelo linear misto com ênfase na ordenação de médias de tratamentos genéticos. Scientia Agricola, v.58, p.109-117, 2001. Disponível em: <http://www.scielo.br/scielo.php?script=sci_arttext\&pid=S010390162001000100017\&lng=en\&nrm=iso $>$. Acesso em: 24 jul. 2009. doi: $10.1590 / \mathrm{S} 0103-90162001000100017$.

DUARTE, J.B.; VENCOVSKY, R. Spatial statistical analysis and selection of genotypes in plant breeding. Pesquisa Agropecuária Brasileira, v.40, p.107-114, 2005. Disponível em: <http://webnotes.sct.embrapa.br/ pab/pab.nsf/ecd 4ca3 ff 88 efcfa 032564 cd 004 ea 083 / 82605d05df46313f83256fc400459826?OpenDocument>. Acesso em: 24 jul. 2009.

DUARTE, J.B. et al. Estimadores de componentes de variância em delineamento de blocos aumentados com tratamentos novos de uma ou mais populações. Pesquisa Agropecuária Brasileira, v.36, p.1155-1167, 2001. Disponível em: <http:/ / w e bnotes.s ct.e mbrapa.br/pab/pab.ns f/ e c d 4 c a 3 f f 88 e fc fa 032564 c d 004 e a 083 / c2192be5d5fd4ba603256ad40059f926? OpenDocument $>$. Acesso em: 24 jul. 2009.

FEDERER,W.T. Augmented (hoonuiaku) designs. Hawaian Planters' Record, Aica, v.55, p.191-208, 1956.

GOMES, F.P. Curso de estatística experimental. 12.ed. Piracicaba: ESALQ/USP, 1987. 467p.

HOGARTH, D.M; ALLSOPP, P.G Manual of canegrowing. Indooroopilly: BSES, 2000. 463p.
MATSUOKA, S. et al. Melhoramento da cana-de-açúcar. In: BORÉM, A. (Ed.). Melhoramento de espécies cultivadas. Viçosa: UFV, 2005. p.225-274.

PETERNELLI, L.A.; BARBOSA, M.H.P. Desafios na condução e planejamento de experimentos de avaliação de clones de canade-açúcar. In: SIMPÓSIO DE ATUALIZAÇÃO EM GENÉTICA E MELHORAMENTO DE PLANTAS: DESAFIOS E PERSPECTIVAS DA INTERAÇÃO DA BIOMETRIA E O MELHORAMENTO DE PLANTAS, 7., 2004, Lavras, MG. Anais... Lavras: UFLA, 2004. V.1, p.17-27. Disponível em: $<$ http://www.nucleoestudo.ufla.br/gen/Paginas/Simposios.htm>. Acesso em: 25 Jul. 2009.

R DEVELOPMENT CORE TEAM. R: A language and environment for statistical computing. $R$ Foundation for Statistical Computing, Vienna, Austria, 2008. Acesso em: 10 Ago. 2008 Online. Disponível na Internet: http://www.Rproject.org.

RAMALHO, M.A.P. et al. Experimentação em genética e melhoramento de plantas. Lavras: UFLA, 2000. 303p.

RESENDE, M.D.V.; BARBOSA, M.H.P. Selection via simulated individual BLUP based on family genotypic effects in sugarcane. Pesquisa Agropecuária Brasileira, v.41, p.421-429, 2006. Disponível em: <http://webnotes.sct.embrapa.br/pab/pab.nsf/ e c d 4 c a 3 ff 88 e f c fa 032564 cd 004 e a 083 / ab33f30228c493038325715500619163? OpenDocument $>$. Acesso em: 24 jul. 2009.

SANTOS, A.H. et al. Simulation of mixed models in augmented block design. Scientia Agricola, v.59, p.483-489, 2002. Disponível em: $<$ http://www.scielo.br/scielo.php?script=sci_arttext\&pid=S010390162002000300012\&lng=en\&nrm=iso>. Acesso em: 24 Jul. 2009. doi: 10.1590/S0103-90162002000300012.

SCOTT, R.A.; MILLIKEN, G.A. A SAS program for analyzing augmented randomized complete-block designs. Crop Science, v.33, p.865-867, 1993.

SOKAL, R.R.; ROHLF, F.J. Biometry - the principle and practice of statistics in biological research. 2.ed. San Francisco: W.H. Freeman, 1981. 859p.

SOUZA, E.F.M. et al. Designs and model effects definitions in the initial stage of a plant breeding program. Pesquisa Agropecuária Brasileira, v.41, p.369-375, 2006. Disponível em: <http://webnotes.sct.embrapa.br/ $\mathrm{pab} / \mathrm{pab} . \mathrm{nsf} / \mathrm{ecd} 4 \mathrm{ca} 3 \mathrm{ff} 88 \mathrm{efcfa} 032564 \mathrm{~cd} 004$ ea $083 /$ f253881c072d0acd832571550059a37e?OpenDocument>. Acesso em: 24 jul. 2009.

SOUZA. A.P. et al. Alternativas experimentais na avaliação de famílias em programas de melhoramento genético do feijoeiro. Pesquisa Agropecuária Brasileira, v.35, p.1765-1771, 2000. Disponível em: <http://webnotes.sct.embrapa.br/pab/ pab.nsf/ecd 4 ca 3 ff 88 efcfa 032564 cd 004 ea 083 / 92c8b12c7cf5bd740325699a006b0929?OpenDocument $>$. Acesso em: 24 jul. 2009.

SOUZA. A.P. et al. Experimental alternatives for evaluation of progenies and clones in eucalyptus breeding programs. Revista Árvore, v.27, p.427-434, 2003. Disponível em: <http:// www.scielo.br/scielo.php?script $=$ sci_arttext\&pid $=\mathrm{S} 0100$ $67622003000400002 \& \operatorname{lng}=\mathrm{en} \& \mathrm{nrm}=\mathrm{iso}>$. Acesso em: $24 \mathrm{Jul}$. 2009. doi: 10.1590/S0100-67622003000400002. 